\title{
Low scale type I seesaw model for lepton masses and mixings
}

\author{
A. E. Cárcamo Hernández, ${ }^{*}$ Marcela González $\bullet{ }^{\dagger}$ and Nicolás A. Neill ${ }^{\ddagger}$ \\ Universidad Técnica Federico Santa María and Centro Científico-Tecnológico de Valparaíso, \\ Casilla 110-V, Valparaíso, Chile
}

(Received 5 June 2019; accepted 15 January 2020; published 6 February 2020)

\begin{abstract}
In contrast to the original type I seesaw mechanism that requires right-handed Majorana neutrinos at energies much higher than the electroweak scale, the so-called low scale seesaw models allow lighter masses for the additional neutrinos. Here we propose an alternative low scale type I seesaw model, where neither linear nor inverse seesaw mechanisms take place, but the spontaneous breaking of a discrete symmetry at an energy scale much lower than the model cutoff is responsible for the smallness of the light active neutrino masses. In this scenario, the model is defined with minimal particle content, where the righthanded Majorana neutrinos can have masses at the $\sim 50 \mathrm{GeV}$ scale. The model is predictive in the neutrino sector having only four effective parameters that allow to successfully reproduce the experimental values of the six low energy neutrino observables.
\end{abstract}

DOI: 10.1103/PhysRevD.101.035005

\section{INTRODUCTION}

After minimally extending the Standard Model (SM) to include massive neutrinos, the observed fermion mass hierarchy is extended over a range of 13 orders of magnitude, from the lightest active neutrino mass scale up to the top quark mass. In addition, the small quark mixing angles decrease from one generation to the next while in the lepton sector this hierarchy is not present since two of the mixing angles are large and the other one is small. Neither of these features in the flavor sector is explained in the SM. This is the so-called SM flavor puzzle, which has motivated the construction of theories with extended scalar and/or fermion sectors with additional continuous or discrete groups. In particular, extensions of the SM with non-Abelian discrete flavor symmetries are very attractive since they successfully describe the observed pattern of fermion masses and mixings (for recent reviews on discrete flavor groups see Refs. [1-6]), while they can naturally appear from the breaking of continuous non-Abelian gauge symmetries or from compactified extra dimensions (see Ref. [7] and references therein). Several discrete groups have been employed in extensions of the SM. In particular, $A_{4}$ is the smallest discrete group with one three-dimensional and

\footnotetext{
*antonio.carcamo@usm.cl

†marcela.gonzalezp@usm.cl

*nicolas.neill@gmail.com
}

Published by the American Physical Society under the terms of the Creative Commons Attribution 4.0 International license. Further distribution of this work must maintain attribution to the author(s) and the published article's title, journal citation, and DOI. Funded by SCOAP. three distinct one-dimensional irreducible representations where the three families of fermions can be accommodated rather naturally. This group has been particularly promising in providing a predictive description of the current pattern of SM fermion masses and mixing angles [8-47]. Despite several models based on the $A_{4}$ discrete symmetry have been proposed, most of them have a nonminimal scalar sector, composed of several $S U(2)$ Higgs doublets, even in their low energy limit, and have $A_{4}$ scalar triplets in the scalar spectrum whose vacuum expectation value (VEV) configurations in the $A_{4}$ direction are not the most natural solutions of the scalar potential minimization equations. Thus, it would be desirable to build an $A_{4}$ flavor model which at low energies reduces to the SM model and where the different gauge singlet scalars are accommodated into $A_{4}$ singlets and one $A_{4}$ triplet [with VEV pattern in the $(1,1,1) A_{4}$ direction] which satisfies the minimization condition of the scalar potential for the whole range of values of the parameter space. To this end, in this work we propose an extension of the SM based on the $A_{4}$ family symmetry, which is supplemented by a $Z_{4}$ auxiliary symmetry, whose spontaneous breaking at an energy scale $\left(v_{S}\right)$ much lower than the model cutoff $(\Lambda)$ produces the small light active neutrino mass scale $m_{\nu}$. As we will show in the next sections, in this scenario the masses for the active neutrinos are produced by a type I seesaw mechanism [48-51] mediated by three $\sim 50 \mathrm{GeV}$ right-handed Majorana neutrinos, where $m_{\nu} \propto\left(v_{S} / \Lambda\right)^{2}$. Given the low mass scale of the right-handed neutrinos, this model can be classified as a low scale type I seesaw, as it has been coined in the literature [52-62]. There are different realizations of low scale seesaw models, as for example inverse or linear [35,46,63-78], where an additional lepton number violating mass parameter is added. 
In these models, the smallness of $m_{\nu}$ is related to the smallness of the additional parameter. In our case, however, no extra small mass parameter has been included, and the smallness of the light neutrino masses is explained through the spontaneous breaking of the auxiliary discrete groups, which leads to a suppression in the Dirac neutrino mass matrix.

From the point of view of the low energy neutrino observables, the model makes very particular predictions for $\delta_{C P}$ and $\theta_{23}$, which are not aligned with the central values of current fits. Therefore, future improvements in the precision of neutrino measurements will provide an experimental test of the model. Processes like (i) charged lepton flavor violating decays $\left(\ell \rightarrow \ell^{\prime} \gamma\right)$ [11,12], (ii) flavor changing neutral currents, and (iii) rare top quark decays such as $t \rightarrow h c, t \rightarrow c Z$ [15], are strongly suppressed, in contrast to other $A_{4}$ flavor models (that usually have several Higgs doublets), where these processes can have rates that are at the reach of forthcoming experiments.

The paper is organized as follows. In Sec. II we describe the model. In Sec. III we present a discussion on lepton masses and mixings and give the corresponding results. We draw our conclusions in Sec. IV. The Appendix provides a concise description of the $A_{4}$ discrete group.

\section{MODEL DESCRIPTION}

We propose an extension of the SM where the scalar sector is augmented by the inclusion of four gauge-singlet scalar fields and the SM gauge symmetry is supplemented by the $A_{4} \times Z_{4}$ discrete group. The symmetry $\mathcal{G}$ features the following spontaneous symmetry breaking pattern:

$$
\begin{gathered}
\mathcal{G}=S U(2)_{L} \times U(1)_{Y} \times A_{4} \times Z_{4} \stackrel{v_{S}}{\rightarrow}, \\
S U(2)_{L} \times U(1)_{Y} \stackrel{v}{\rightarrow} U(1)_{Q},
\end{gathered}
$$

where the symmetry-breaking scales satisfy the hierarchy $v_{S} \sim \mathcal{O}(1) \mathrm{TeV}>v, v_{S}$ is the scale of spontaneous breaking of the $A_{4} \times Z_{4}$ discrete group, and $v=246 \mathrm{GeV}$ is the electroweak symmetry breaking scale. As mentioned before, the scalar sector of the SM is augmented by the inclusion of four SM gauge singlet scalars. We add these extra scalar fields for the following reasons: (i) to build nonrenormalizable charged leptons and Dirac neutrino Yukawa terms invariant under the local and discrete groups, crucial to generate predictive textures for the lepton sector; (ii) to generate a renormalizable Yukawa term for the righthanded Majorana neutrinos, that can give rise to $\sim 50 \mathrm{GeV}$ masses for these singlet fermions. As we will see below, the observed pattern of SM charged lepton masses and leptonic mixing angles will arise from the spontaneous breaking of the $A_{4} \times Z_{4}$ discrete group. In order to generate the masses for the light active neutrinos via a type I seesaw mechanism, we extend the fermion sector by including three righthanded Majorana neutrinos, which are singlets under the
SM group. The lepton assignments under the group $A_{4} \times Z_{4}$ are

$$
\begin{aligned}
L_{L} & \sim(\mathbf{3}, 0), & N_{R} & \sim(\mathbf{3}, 1), \\
l_{1 R} & \sim\left(\mathbf{1}^{\prime}, 1\right), & & l_{2 R} \sim(\mathbf{1}, 1),
\end{aligned}
$$

Here we specify the dimensions of the $A_{4}$ irreducible representations by the numbers in boldface and we write the different $Z_{4}$ charges in additive notation. Regarding the lepton sector, note that the left- and right-handed leptonic fields are accommodated into $A_{4}$ triplet and $A_{4}$ singlet irreducible representations, respectively, whereas the righthanded Majorana neutrinos are unified into an $A_{4}$ triplet. The scalar spectrum of the model includes the SM Higgs doublet $\phi$ and the gauge singlet scalars $\rho, \xi_{j}(j=1,2,3)$. The scalar fields have the following transformation properties under the flavor symmetry $A_{4} \times Z_{4}$ :

$\phi \sim(\mathbf{1}, 0), \quad \rho_{1} \sim\left(\mathbf{1}^{\prime \prime},-1\right), \quad \rho_{2} \sim\left(\mathbf{1}^{\prime},-1\right)$,

$\rho_{3} \sim(\mathbf{1},-2), \quad \xi \sim(\mathbf{3},-1)$.

We assume the following vacuum configuration for the $A_{4}$-triplet gauge singlet scalar $\xi$ :

$$
\langle\xi\rangle=\frac{v_{\xi}}{\sqrt{3}}(1,1,1),
$$

which satisfies the minimization condition of the scalar potential for the whole range of values of the parameter space, as shown in Ref. [31]. With the particle content previously specified, we have the following relevant Yukawa terms for the lepton sector, invariant under the symmetries of the model:

$$
\begin{aligned}
\mathcal{L}_{Y}^{(L)}= & y_{1}^{(L)}\left(\bar{L}_{L} \phi \xi\right)_{\mathbf{1}^{\prime \prime}} l_{1 R} \frac{1}{\Lambda}+y_{2}^{(L)}\left(\bar{L}_{L} \phi \xi\right)_{\mathbf{1}} l_{2 R} \frac{1}{\Lambda} \\
& +y_{3}^{(L)}\left(\bar{L}_{L} \phi \xi\right)_{\mathbf{1}^{\prime}} l_{3 R} \frac{1}{\Lambda}+y_{N}\left(\bar{N}_{R} N_{R}^{C}\right)_{\mathbf{1}}\left(\rho_{3}+c \rho_{3}^{*}\right) \\
& +y_{1 \nu}\left(\bar{L}_{L} \tilde{\phi} N_{R}\right)_{\mathbf{1}^{\prime}} \frac{\rho_{1}}{\Lambda}+y_{2 \nu}\left(\bar{L}_{L} \tilde{\phi} N_{R}\right)_{\mathbf{1}^{\prime \prime}} \frac{\rho_{2}}{\Lambda} \\
& +y_{3 \nu}\left(\bar{L}_{L} \tilde{\phi} N_{R}\right)_{\mathbf{3}} \frac{\xi}{\Lambda}+y_{4 \nu}\left(\bar{L}_{L} \tilde{\boldsymbol{\phi}} N_{R}\right)_{\mathbf{3} \mathbf{a}} \frac{\xi}{\Lambda}+\text { H.c. },
\end{aligned}
$$

where the dimensionless couplings in Eq. (5) are $\mathcal{O}(1)$ parameters.

In what follows, we describe the role of each discrete group factor of our $A_{4}$ flavor model. The $A_{4}$ discrete group yields a reduction of the number of model parameters, giving rise to predictive textures for the lepton sector, which are consistent with the lepton mass and mixing pattern, as will be shown in Sec. III. On the other hand, the $Z_{4}$ discrete group is the smallest cyclic symmetry allowing a renormalizable Yukawa term for the right-handed Majorana neutrinos, giving rise to a diagonal Majorana neutrino 
mass matrix that yields degenerate Majorana neutrinos with electroweak scale masses. In addition, the spontaneous breaking of the $A_{4} \times Z_{4}$ discrete group at an energy scale much lower than the model cutoff is crucial to produce small mixing mass terms between the active and sterile neutrinos, allowing the implementation of a low scale type I seesaw mechanism. Finally, we assume that the VEVs of the gauge singlet scalar fields $\xi, \rho_{i}(i=1,2,3)$ satisfy the relation

$$
v_{\rho_{j}} \sim v_{\xi} \sim \mathcal{O}(1) \mathrm{TeV} \ll \Lambda, \quad j=1,2,3,
$$

where $v_{\xi} \sim v_{\rho} \sim v_{S}$ is the discrete symmetry breaking scale and $\Lambda$ is the model cutoff.

It is worth mentioning that this model at low energies corresponds to a singlet-doublet model $[79,80]$. Consequently, from a detailed analysis of the low energy scalar potential (as done for example in Ref. [81]) one can show that the $125 \mathrm{GeV}$ SM-like Higgs boson has couplings close to the SM expectation, with small deviations of order $v^{2} / v_{S}^{2} \sim \mathcal{O}\left(10^{-2}\right)$. The TeV-scale singlet $s^{0}\left(s^{0}=\xi, \rho_{j}\right)$ will mix with the $C P$-even neutral component of the SM Higgs doublet, $h^{0}$, with a mixing angle $\gamma \sim \mathcal{O}\left(v / v_{S}\right)$. Thus, the couplings of the singlet scalars to the SM particles will be equal to the SM Higgs couplings times the $s^{0}-h^{0}$ mixing angle $\gamma$. The collider phenomenology of this scenario is well studied [82-86]. For $\mathrm{TeV}$-scale singlets, the most stringent limits at the $8 \mathrm{TeV}$ LHC come from indirect searches. A global fit to all SM signal strengths constrains $\sin ^{2} \gamma \leq 0.23$ at 95\% C.L. $[87,88]$, that assuming $\mathcal{O}(1)$ couplings in the scalar potential translates to $v_{S} \gtrsim 500 \mathrm{GeV}$. For a summary of the sensitivity of future colliders see for example Table 1 of Ref. [86]. As we will see in the next section, there is a broad range of values of $v_{S}$ that are consistent with the observed light neutrino masses and current limits on singlet scalars.

\section{NEUTRINO MASSES AND MIXINGS}

The lepton Yukawa terms in Eq. (5) imply that the mass matrix for charged leptons is given by

$$
M_{l}=V_{l L} S_{\ell} \operatorname{diag}\left(m_{e},-m_{\mu}, m_{\tau}\right),
$$

where

$$
V_{l L}=\frac{1}{\sqrt{3}}\left(\begin{array}{ccc}
1 & 1 & 1 \\
1 & \omega^{2} & \omega \\
1 & \omega & \omega^{2}
\end{array}\right), \quad S_{\ell}=\left(\begin{array}{ccc}
0 & -1 & 0 \\
1 & 0 & 0 \\
0 & 0 & 1
\end{array}\right), \quad \omega=e^{\frac{2 \pi i}{3}}
$$

SO

$$
M_{l}=\frac{1}{\sqrt{3}}\left(\begin{array}{ccc}
m_{e} & m_{\mu} & m_{\tau} \\
\omega^{2} m_{e} & m_{\mu} & \omega m_{\tau} \\
\omega m_{e} & m_{\mu} & \omega^{2} m_{\tau}
\end{array}\right)
$$

Regarding the neutrino sector, we find that the resulting Dirac neutrino mass matrix reads

$M_{\nu}^{D}=\left(\begin{array}{ccc}b+c & a+d & a-d \\ a-d & \omega b+c \omega^{2} & a+d \\ a+d & a-d & \omega^{2} b+c \omega\end{array}\right) \frac{v v_{S}}{\sqrt{2} \Lambda}$,

where $\omega=e^{\frac{2 \pi i}{3}}$ and $a, b, c, d$ are effective parameters related to the neutrino Yukawa couplings in Eq. (5).

The fermion sector is extended by including three righthanded Majorana neutrinos with masses $m_{N}$, where the Majorana mass matrix $M_{N}$ is proportional to the identity, $M_{N}=m_{N} \hat{\mathbf{1}}_{3 \times 3}$. Given that $m_{N} \gg\left(M_{\nu}^{D}\right)_{i j}(i, j=1,2,3)$, the light active neutrino mass matrix $\left(M_{\nu}\right)$ arises from a type I seesaw mechanism:

$$
\begin{aligned}
M_{\nu}= & M_{\nu}^{D} M_{N}^{-1}\left(M_{\nu}^{D}\right)^{T} \\
= & \frac{1}{m_{N}}\left(\begin{array}{ccc}
b+c & a+d & a-d \\
a-d & \omega b+c \omega^{2} & a+d \\
a+d & a-d & \omega^{2} b+c \omega
\end{array}\right) \\
& \times\left(\begin{array}{ccc}
b+c & a-d & a+d \\
a+d & c \omega^{2}+b \omega & a-d \\
a-d & a+d & b \omega^{2}+c \omega
\end{array}\right) \frac{v^{2} v_{S}^{2}}{2 \Lambda^{2}},
\end{aligned}
$$

where we can read that the typical mass scale of the light active neutrinos is

$$
m_{\nu} \sim \frac{v^{2}}{2 m_{N}}\left(\frac{v_{S}}{\Lambda}\right)^{2}
$$

It is noteworthy that the smallness of the active neutrino masses is a consequence of their inverse scaling with the square of the model cutoff, which is much larger than the breaking scale $\left(v_{S}\right)$ of the discrete symmetries. We can see from Eq. (13) that for heavy neutrinos with masses $m_{N} \sim \mathcal{O}(50 \mathrm{GeV})$, there is a wide range of values of $v_{S}$ that produce the required suppression, depending on the specific value of the model cutoff. To show that the model is consistent with the neutrino oscillation experimental data, we fix $m_{\nu}=50 \mathrm{meV}$ and vary the neutrino sector parameters $a, b, c$ and $d$, to adjust the neutrino mass squared splittings $\Delta m_{21}^{2}, \Delta m_{31}^{2}$, the leptonic mixing angles $\theta_{12}, \theta_{13}, \theta_{23}$, and the leptonic Dirac $C P$ violating phase $\delta_{C P}$ to their experimental values.

Tables I and II show the average model values and experimental values of the neutrino observables for both 
TABLE I. Normal mass hierarchy.-Model and experimental values of the neutrino mass squared splittings, leptonic mixing angles, and $C P$-violating phase. The second column shows the average model value for each observable, calculated from the model solutions that reproduce the neutrino observables at the $90 \%$ C.L. The experimental values are taken from Refs. [89,90].

\begin{tabular}{|c|c|c|c|c|c|}
\hline \multirow[b]{2}{*}{ Observable } & \multirow{2}{*}{$\begin{array}{c}\text { Average } \\
\text { model value }\end{array}$} & \multicolumn{4}{|c|}{ Neutrino oscillation global fit values $(\mathrm{NH})$} \\
\hline & & Best fit $\pm 1 \sigma[89]$ & Best fit $\pm 1 \sigma[90]$ & $3 \sigma$ range $[89]$ & $3 \sigma$ range $[90]$ \\
\hline$\Delta m_{21}^{2}\left[10^{-5} \mathrm{eV}^{2}\right]$ & 7.61 & $7.55_{-0.16}^{+0.20}$ & $7.39_{-0.20}^{+0.21}$ & $7.05-8.14$ & $6.79-8.01$ \\
\hline$\Delta m_{31}^{2}\left[10^{-3} \mathrm{eV}^{2}\right]$ & 2.51 & $2.50 \pm 0.03$ & $2.525_{-0.032}^{+0.033}$ & $2.41-2.60$ & $2.427-2.625$ \\
\hline$\theta_{12}\left({ }^{\circ}\right)$ & 34.1 & $34.5_{-1.0}^{+1.2}$ & $33.82_{-0.76}^{+0.032}$ & $31.5-38.0$ & $31.61-36.27$ \\
\hline$\theta_{13}\left({ }^{\circ}\right)$ & 8.45 & $8.45_{-0.14}^{+0.16}$ & $8.61 \pm 0.13$ & $8.0-8.9$ & $8.22-8.99$ \\
\hline$\theta_{23}\left({ }^{\circ}\right)$ & 42.8 & $47.7_{-1.7}^{+1.2}$ & $49.6_{-12}^{+1.0}$ & $41.8-50.7$ & $40.3-52.4$ \\
\hline$\delta_{C P}\left({ }^{\circ}\right)$ & 313 & $218_{-27}^{+38}$ & $215_{-29}^{+40}$ & $157-349$ & $125-392$ \\
\hline
\end{tabular}

TABLE II. Inverted mass hierarchy.--Model and experimental values of the neutrino mass squared splittings, leptonic mixing angles, and $C P$-violating phase. The second column shows the average model value for each observable, calculated from the model solutions that reproduce the neutrino observables at the $90 \%$ C.L. The experimental values are taken from Refs. [89,90].

\begin{tabular}{|c|c|c|c|c|c|}
\hline \multirow[b]{2}{*}{ Observable } & \multirow{2}{*}{$\begin{array}{c}\text { Average } \\
\text { model value }\end{array}$} & \multicolumn{4}{|c|}{ Neutrino oscillation global fit values (IH) } \\
\hline & & Best fit $\pm 1 \sigma$ [89] & Best fit $\pm 1 \sigma[90]$ & $3 \sigma$ range $[89]$ & $3 \sigma$ range [90] \\
\hline$\Delta m_{21}^{2}\left[10^{-5} \mathrm{eV}^{2}\right]$ & 7.61 & $7.55_{-0.16}^{+0.20}$ & $7.39_{-0.20}^{+0.21}$ & $7.05-8.14$ & $6.79-8.01$ \\
\hline$\Delta m_{13}^{2}\left[10^{-3} \mathrm{eV}^{2}\right]$ & 2.41 & $2.42_{-0.04}^{+0.03}$ & $2.512_{-0.032}^{+0.034}$ & $2.31-2.51$ & $2.412-2.611$ \\
\hline$\theta_{12}\left(^{\circ}\right)$ & 34.7 & $34.5_{-1.0}^{+1.2}$ & $33.82_{-0.76}^{+0.078}$ & $31.5-38.0$ & $31.61-36.27$ \\
\hline$\theta_{13}\left({ }^{\circ}\right)$ & 8.56 & $8.53_{-0.15}^{+0.14}$ & $8.65 \pm 0.13$ & $8.1-9.0$ & $8.27-9.03$ \\
\hline$\theta_{23}\left({ }^{\circ}\right)$ & 48.7 & $47.9_{-1 .}^{+1.0}$ & $49.8_{-11}^{+1.0}$ & $42.3-50.7$ & $40.6-52.5$ \\
\hline$\delta_{C P}\left({ }^{\circ}\right)$ & 297 & $281_{-27}^{+23}$ & $284_{-29}^{+27}$ & $202-349$ & $196-360$ \\
\hline
\end{tabular}

normal hierarchy $(\mathrm{NH})$ and inverted hierarchy (IH). Figure 1 shows several solutions consistent with the global fits for the $\mathrm{NH}$ (the $\mathrm{IH}$ has the same behavior). The dots in orange correspond to the model values, which
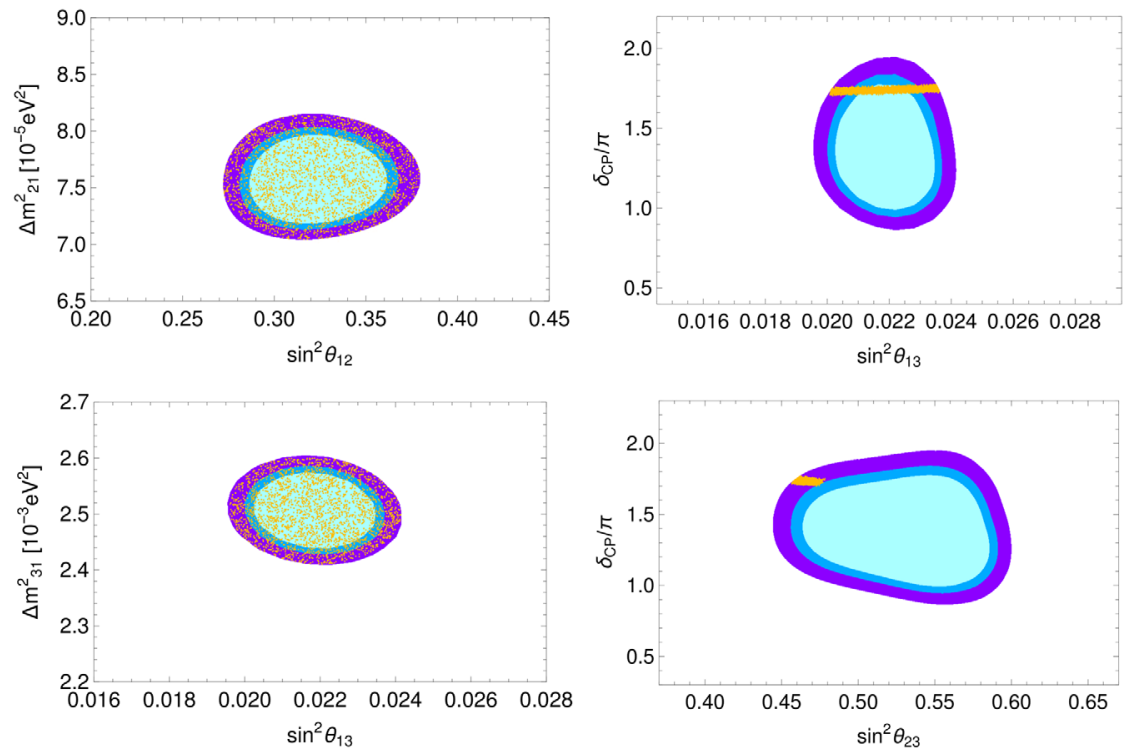

FIG. 1. Correlation between the observables predicted by the model: $\sin ^{2} \theta_{12}, \sin ^{2} \theta_{13}, \sin ^{2} \theta_{23}, \delta_{C P}, \Delta m_{21}^{2}$, and $\Delta m_{31}^{2}$ for the normal hierarchy, superimposed on the global fits from Ref. [89]. Model predictions are shown in orange, while the 90\%, 95\%, and 99\% C.L. contours of the global fit are in purple, blue, and light blue, respectively. 
$a \simeq-0.474, \quad b \simeq-0.367, \quad c \simeq 0.487, \quad d \simeq-0.0590(\mathrm{NH})$

$a \simeq-0.254, \quad b \simeq 0.352, \quad c \simeq-0.795, \quad d \simeq 0.0174(\mathrm{IH})$

which produces the following mass spectrum:

$$
\begin{array}{lll}
m_{1} \simeq 5.84[\mathrm{meV}], & m_{2} \simeq 10.5[\mathrm{meV}], & m_{3} \simeq 50.4[\mathrm{meV}](\mathrm{NH}) \\
m_{1} \simeq 50.4[\mathrm{meV}], & m_{2} \simeq 51.1[\mathrm{meV}], & m_{3} \simeq 11.7[\mathrm{meV}](\mathrm{IH}) .
\end{array}
$$

Thus, with four effective parameters, the model reproduces the experimental values of the six physical observables of the neutrino sector, i.e., the neutrino mass squared splittings $\Delta m_{21}^{2}$ and $\Delta m_{31}^{2}$, the leptonic mixing angles $\theta_{12}, \theta_{23}, \theta_{13}$, and the leptonic Dirac $C P$ violating phase $\delta_{C P}$. The model values are consistent with the current neutrino oscillation experimental data, for both normal and inverted mass ordering, as shown in Tables I and II. From Fig. 1, we can see that for the normal hierarchy, $\Delta m_{31}^{2}, \Delta m_{21}^{2}, \sin ^{2} \theta_{12}$, and $\sin ^{2} \theta_{13}$ are evenly distributed in the allowed range. On the other hand, for $\sin ^{2} \theta_{23}$ and $\delta_{C P}$, the model features more definite predictions. The same behavior is found for the inverted hierarchy.

It is worth mentioning that in a generic scenario, the neutrino Yukawa couplings are complex, thus the light active neutrino sector has eight parameters. However, not all of them are physical. Considering the case of real VEVs for the gauge-singlet scalars $\rho_{1}, \rho_{2}$, and $\xi$, the phase redefinition of the leptonic fields $L_{L}$ and $N_{R}$ allows to rotate away the phase of one of the neutrino Yukawa couplings, leading to seven physical parameters. On the other hand, if we consider complex VEVs for the gaugesinglet scalars $\rho_{1}, \rho_{2}$, and $\xi$, we can use their phases to set three of the four neutrino Yukawa couplings real. Therefore, in this case we are left with five effective parameters in the neutrino sector. However, for the sake of simplicity, we are considering a particular benchmark scenario with real neutrino Yukawa couplings, i.e., four effective parameters. In this simplified benchmark scenario, the complex phase responsible for $C P$ violation in neutrino oscillation arises from the spontaneous breaking of the $A_{4}$ discrete group. This mechanism for inducing $C P$ violation in the fermion sector via the spontaneous breaking of discrete groups is the so-called geometrical $C P$ violation [46,75,76,91-97].

Now we determine the effective Majorana neutrino mass parameter, which is proportional to the neutrinoless double beta decay $(0 \nu \beta \beta)$ amplitude. The effective Majorana neutrino mass parameter is given by

$$
m_{\beta \beta}=\left|\sum_{j} m_{\nu_{j}} U_{e j}^{2}\right|,
$$

where $U_{e j}^{2}$ and $m_{\nu_{j}}$ are the Pontecorvo-Maki-NakagawaSakata mixing matrix elements and the Majorana neutrino masses, respectively. As we can see from Fig. 2, the predicted effective Majorana neutrino mass parameter is within the range:

$$
\begin{aligned}
& m_{\beta \beta}=(5.0-7.4) \mathrm{meV} \quad(\mathrm{NH}), \\
& m_{\beta \beta}=(24.7-35.3) \mathrm{meV} \quad(\mathrm{IH}),
\end{aligned}
$$

which is below the sensitivity of present $0 \nu \beta \beta$-decay experiments. The current experimental sensitivity on the Majorana neutrino mass parameter is obtained from the KamLANDZen limit on the ${ }^{136} \mathrm{Xe} 0 \nu \beta \beta$ decay half-life, $T_{1 / 2}^{0 \nu \beta \beta}\left({ }^{136} \mathrm{Xe}\right) \geq$ $1.07 \times 10^{26} \mathrm{yr}$ [98], which yields the corresponding upper limit on the Majorana mass, $\left|m_{\beta \beta}\right| \leq(61-165) \mathrm{meV}$ at $90 \%$ C.L. For other $0 \nu \beta \beta$-decay experiments see Refs. [99104]. The experimental sensitivity of neutrinoless double beta decay searches is expected to improve in the near future. Note that the model predicts a range of values for neutrinoless double beta decay rates that can be tested by the nextgeneration bolometric CUORE experiment [102], as well as the next-to-next-generation ton-scale $0 \nu \beta \beta$-decay experiments $[98,101,105,106]$.

Finally, we briefly comment on the prospects of observing heavy neutrinos with masses around $50 \mathrm{GeV}$ in collider experiments. In the type I seesaw model, the heavy light mixing squared, $|U|^{2}$ is given by

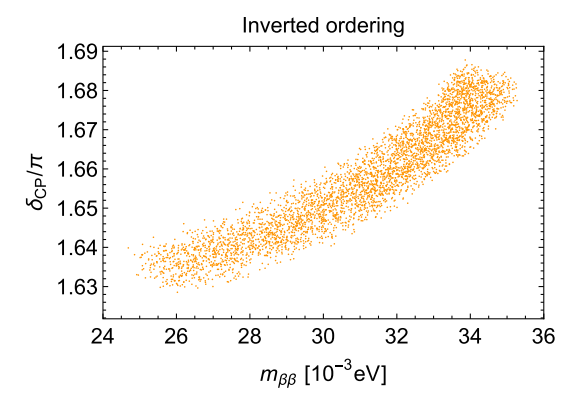

FIG. 2. Model predictions for the Dirac $C P$ violating phase versus the effective Majorana mass parameter. 


$$
|U|^{2} \sim\left(\frac{M_{D}}{M_{N}}\right)^{2} \sim \frac{m_{\nu}}{m_{N}}
$$

which in our case (for $m_{N} \approx 50 \mathrm{GeV}$ and $m_{\nu} \approx 50 \mathrm{meV}$ ) gives $|U|^{2} \sim O\left(10^{-12}\right)$. Even though this is a very small mixing, typical of the type I seesaw model, for masses $m_{N} \sim 50 \mathrm{GeV}$ it might be within the reach of future colliders such as the FCC-ee [107]. The most sensitive channel at the FCC-ee would be $Z \rightarrow \nu N$, when the decays of $N$ are fully reconstructible, i.e., $N \rightarrow \ell W^{*} \rightarrow \ell q \bar{q}^{\prime}$. According to the analysis in Ref. [107], most of the backgrounds for this decay can be reduced if one takes into consideration (i) the displaced vertex topology produced by the long-lived $N$ (expected for these small couplings) and (ii) the full reconstruction of the heavy neutrino mass, allowed by its visible decay. For $\sim 10^{13} \mathrm{Z}$ decays, this would allow reaching sensitivities down to a heavy-light mixing $|U|^{2} \sim 10^{-12}$, for heavy neutrino masses between 40 and $80 \mathrm{GeV}$.

\section{SUMMARY AND CONCLUSIONS}

We have proposed a low scale seesaw model based on the $A_{4} \times Z_{4}$ discrete symmetry, where the masses for the light active neutrinos are produced by a type I seesaw mechanism mediated by three $\sim 50 \mathrm{GeV}$ scale right-handed Majorana neutrinos. Contrary to the original type I seesaw, where the right-handed neutrinos are required to have masses much larger than the electroweak scale to reproduce the light active neutrino mass scale $m_{\nu}$, in this case $m_{\nu}$ is suppressed by the ratio between the discrete symmetry breaking scale $\left(v_{S}\right)$ and the cutoff $(\Lambda)$ of the model: $m_{\nu} \propto\left(v_{S} / \Lambda\right)^{2}$. That is, the large $\Lambda / v_{S}$ ratio plays the role of the heavy mass scale in the original seesaw. This allows lighter Majorana masses that might be eventually tested at future colliders such as the FCC-ee.

The model is predictive in the sense that it reproduces the experimental values of the six low energy neutrino observables with only four effective parameters. Two of the predicted observables (being within the $90 \%$ C.L. global-fit regions) are not aligned with the central values of the global fits, so are distinctive predictions of the model. These are the $C P$-violating angle, predicted to be

$$
\delta_{C P}= \begin{cases}312.9^{\circ} \pm 2.4^{\circ}, & (\mathrm{NH}) \\ 297.2^{\circ} \pm 2.7^{\circ}, & (\mathrm{IH}),\end{cases}
$$

and the "atmospheric" neutrino mixing angle

$$
\sin ^{2} \theta_{23}= \begin{cases}0.465 \pm 0.004, & (\mathrm{NH}) \\ 0.565 \pm 0.001, & (\mathrm{IH}) .\end{cases}
$$

The effective Majorana neutrino mass parameter is predicted to be

$$
m_{\beta \beta}=\left\{\begin{array}{cc}
(6.2 \pm 0.5) \mathrm{meV}, & (\mathrm{NH}) \\
(31.1 \pm 2.6) \mathrm{meV}, & (\mathrm{IH}) .
\end{array}\right.
$$

The scalar sector of the model corresponds to the SM Higgs doublet supplemented with additional singlet scalars. The phenomenology of this kind of extended Higgs sectors is well studied, and many direct and indirect searches have been proposed in the literature. For masses of the additional scalar singlets $\left(m_{S}\right)$ in the range $1 \mathrm{TeV} \lesssim m_{S} \lesssim 11 \mathrm{TeV}$, the scalar sector would be within the reach of future colliders.

\section{ACKNOWLEDGMENTS}

This work was supported by FONDECYT (Chile) under Grants No. 1170803, No. 3170906 and No. 11180873, and in part by Conicyt PIA/Basal FB0821.

\section{APPENDIX: THE PRODUCT RULES FOR $\boldsymbol{A}_{\mathbf{4}}$}

The $A_{4}$ group has one three-dimensional $\mathbf{3}$ and three distinct one-dimensional $\mathbf{1}, \mathbf{1}^{\prime}$, and $\mathbf{1}^{\prime \prime}$ irreducible representations, satisfying the following product rules:

$$
\begin{aligned}
3 \otimes 3 & =3_{s} \oplus 3_{a} \oplus \mathbf{1} \oplus \mathbf{1}^{\prime} \oplus \mathbf{1}^{\prime \prime}, \\
\mathbf{1} \otimes \mathbf{1} & =\mathbf{1}, \quad \mathbf{1}^{\prime} \otimes \mathbf{1}^{\prime \prime}=\mathbf{1}, \\
\mathbf{1}^{\prime} \otimes \mathbf{1}^{\prime} & =\mathbf{1}^{\prime \prime}, \quad \mathbf{1}^{\prime \prime} \otimes \mathbf{1}^{\prime \prime}=\mathbf{1}^{\prime} .
\end{aligned}
$$

Considering $\left(x_{1}, y_{1}, z_{1}\right)$ and $\left(x_{2}, y_{2}, z_{2}\right)$ as the basis vectors for two $A_{4}$-triplets 3 , the following relations are fulfilled:

$$
\begin{aligned}
(\mathbf{3} \otimes \mathbf{3})_{\mathbf{1}} & =x_{1} y_{1}+x_{2} y_{2}+x_{3} y_{3}, \\
(\mathbf{3} \otimes \mathbf{3})_{\mathbf{3}_{s}} & =\left(x_{2} y_{3}+x_{3} y_{2}, x_{3} y_{1}+x_{1} y_{3}, x_{1} y_{2}+x_{2} y_{1}\right), \\
(\mathbf{3} \otimes \mathbf{3})_{\mathbf{1}^{\prime}} & =x_{1} y_{1}+\omega x_{2} y_{2}+\omega^{2} x_{3} y_{3}, \\
(\mathbf{3} \otimes \mathbf{3})_{\mathbf{3}_{a}} & =\left(x_{2} y_{3}-x_{3} y_{2}, x_{3} y_{1}-x_{1} y_{3}, x_{1} y_{2}-x_{2} y_{1}\right), \\
(\mathbf{3} \otimes \mathbf{3})_{\mathbf{1}^{\prime \prime}} & =x_{1} y_{1}+\omega^{2} x_{2} y_{2}+\omega x_{3} y_{3},
\end{aligned}
$$

where $\omega=e^{i \frac{2 \pi}{3}}$. The representation 1 is trivial, while the nontrivial $\mathbf{1}^{\prime}$ and $\mathbf{1}^{\prime \prime}$ are complex conjugate to each other. Reviews of discrete symmetries in particle physics can be found in Refs. [1-4]. 
[1] H. Ishimori, T. Kobayashi, H. Ohki, Y. Shimizu, H. Okada, and M. Tanimoto, Prog. Theor. Phys. Suppl. 183, 1 (2010).

[2] G. Altarelli and F. Feruglio, Rev. Mod. Phys. 82, 2701 (2010).

[3] S. F. King and C. Luhn, Rep. Prog. Phys. 76, 056201 (2013).

[4] S. F. King, A. Merle, S. Morisi, Y. Shimizu, and M. Tanimoto, New J. Phys. 16, 045018 (2014).

[5] S. F. King, Prog. Part. Nucl. Phys. 94, 217 (2017).

[6] S. T. Petcov, Eur. Phys. J. C 78, 709 (2018).

[7] S. F. King, arXiv:1904.06660.

[8] E. Ma and G. Rajasekaran, Phys. Rev. D 64, 113012 (2001).

[9] X. G. He, Y. Y. Keum, and R. R. Volkas, J. High Energy Phys. 04 (2006) 039.

[10] M. C. Chen and S. F. King, J. High Energy Phys. 06 (2009) 072.

[11] F. Feruglio, C. Hagedorn, Y. Lin, and L. Merlo, Nucl. Phys. B832, 251 (2010).

[12] G. Altarelli, F. Feruglio, L. Merlo, and E. Stamou, J. High Energy Phys. 08 (2012) 021

[13] Y. H. Ahn and S. K. Kang, Phys. Rev. D 86, 093003 (2012).

[14] N. Memenga, W. Rodejohann, and H. Zhang, Phys. Rev. D 87, 053021 (2013).

[15] S. Morisi, M. Nebot, K. M. Patel, E. Peinado, and J. W. F. Valle, Phys. Rev. D 88, 036001 (2013).

[16] R. Gonzalez Felipe, H. Serodio, and J. P. Silva, Phys. Rev. D 88, 015015 (2013).

[17] I. de Medeiros Varzielas and D. Pidt, J. High Energy Phys. 03 (2013) 065.

[18] H. Ishimori and E. Ma, Phys. Rev. D 86, 045030 (2012).

[19] S. F. King, S. Morisi, E. Peinado, and J. W. F. Valle, Phys. Lett. B 724, 68 (2013).

[20] A. E. Carcamo Hernandez, I. de Medeiros Varzielas, S. G. Kovalenko, H. Pas, and I. Schmidt, Phys. Rev. D 88, 076014 (2013).

[21] K. S. Babu, E. Ma, and J. W. F. Valle, Phys. Lett. B 552, 207 (2003).

[22] G. Altarelli and F. Feruglio, Nucl. Phys. B741, 215 (2006).

[23] S. Gupta, A. S. Joshipura, and K. M. Patel, Phys. Rev. D 85, 031903 (2012).

[24] G. Altarelli and F. Feruglio, Nucl. Phys. B720, 64 (2005).

[25] A. Kadosh and E. Pallante, J. High Energy Phys. 08 (2010) 115.

[26] A. Kadosh, J. High Energy Phys. 06 (2013) 114.

[27] F. del Aguila, A. Carmona, and J. Santiago, J. High Energy Phys. 08 (2010) 127.

[28] M. D. Campos, A. E. Cárcamo Hernández, S. Kovalenko, I. Schmidt, and E. Schumacher, Phys. Rev. D 90, 016006 (2014).

[29] V. V. Vien and H. N. Long, Int. J. Mod. Phys. A 30, 1550117 (2015).

[30] A. S. Joshipura and K. M. Patel, Phys. Lett. B 749, 159 (2015).

[31] A. E. Cárcamo Hernández and R. Martinez, Nucl. Phys. B905, 337 (2016).

[32] B. Karmakar and A. Sil, Phys. Rev. D 96, 015007 (2017).

[33] D. Borah and B. Karmakar, Phys. Lett. B 780, 461 (2018).
[34] P. Chattopadhyay and K. M. Patel, Nucl. Phys. B921, 487 (2017).

[35] A. E. Cárcamo Hernández and H. N. Long, J. Phys. G 45, 045001 (2018).

[36] E. Ma and G. Rajasekaran, Europhys. Lett. 119, 31001 (2017).

[37] S. Centelles Chuliá, R. Srivastava, and J. W. F. Valle, Phys. Lett. B 773, 26 (2017).

[38] F. Björkeroth, E. J. Chun, and S. F. King, Phys. Lett. B 777, 428 (2018).

[39] R. Srivastava, C. A. Ternes, M. Tórtola, and J. W. F. Valle, Phys. Lett. B 778, 459 (2018).

[40] D. Borah and B. Karmakar, Phys. Lett. B 789, 59 (2019).

[41] A. S. Belyaev, S. F. King, and P. B. Schaefers, Phys. Rev. D 97, 115002 (2018).

[42] A. E. Cárcamo Hernández and S. F. King, Phys. Rev. D 99, 095003 (2019).

[43] R. Srivastava, C. A. Ternes, M. Tórtola, and J. W. F. Valle, Phys. Rev. D 97, 095025 (2018).

[44] L. M. G. De La Vega, R. Ferro-Hernandez, and E. Peinado, Phys. Rev. D 99, 055044 (2019).

[45] S. Pramanick, arXiv:1903.04208.

[46] A. E. Cárcamo Hernández, J. Marchant González, and U. J. Saldana-Salazar, Phys. Rev. D 100, 035024 (2019).

[47] H. Okada and M. Tanimoto, arXiv:1905.13421.

[48] J. Schechter and J. W. F. Valle, Phys. Rev. D 22, 2227 (1980).

[49] J. Schechter and J. W. F. Valle, Phys. Rev. D 25, 774 (1982).

[50] S. F. King, Nucl. Phys. B576, 85 (2000).

[51] S. F. King, J. High Energy Phys. 09 (2002) 011.

[52] J. Gluza, Acta Phys. Pol. B 33, 1735 (2002).

[53] J. Kersten and A. Y. Smirnov, Phys. Rev. D 76, 073005 (2007).

[54] A. Das and N. Okada, Phys. Lett. B 774, 32 (2017).

[55] Z. z. Xing, Prog. Theor. Phys. Suppl. 180, 112 (2009).

[56] X. G. He, S. Oh, J. Tandean, and C. C. Wen, Phys. Rev. D 80, 073012 (2009).

[57] R. Adhikari and A. Raychaudhuri, Phys. Rev. D 84, 033002 (2011).

[58] A. Ibarra, E. Molinaro, and S. T. Petcov, J. High Energy Phys. 09 (2010) 108.

[59] A. Ibarra, E. Molinaro, and S. T. Petcov, Phys. Rev. D 84, 013005 (2011).

[60] J. Lopez-Pavon, E. Molinaro, and S. T. Petcov, J. High Energy Phys. 11 (2015) 030.

[61] C. G. Cely, A. Ibarra, E. Molinaro, and S. T. Petcov, Phys. Lett. B 718, 957 (2013).

[62] C. H. Lee, P. S. Bhupal Dev, and R. N. Mohapatra, Phys. Rev. D 88, 093010 (2013).

[63] R. N. Mohapatra and J. W. F. Valle, Phys. Rev. D 34, 1642 (1986).

[64] M. C. Gonzalez-Garcia and J. W. F. Valle, Phys. Lett. B 216, 360 (1989).

[65] E. K. Akhmedov, M. Lindner, E. Schnapka, and J. W. F. Valle, Phys. Rev. D 53, 2752 (1996).

[66] E. K. Akhmedov, M. Lindner, E. Schnapka, and J. W. F. Valle, Phys. Lett. B 368, 270 (1996).

[67] M. Malinsky, J. C. Romao, and J. W. F. Valle, Phys. Rev. Lett. 95, 161801 (2005). 
[68] P. S. B. Dev and R. N. Mohapatra, Phys. Rev. D 81, 013001 (2010).

[69] A. Abada and M. Lucente, Nucl. Phys. B885, 651 (2014).

[70] C. O. Dib, G. R. Moreno, and N. A. Neill, Phys. Rev. D 90, 113003 (2014).

[71] A. E. Cárcamo Hernández, S. Kovalenko, H. N. Long, and I. Schmidt, J. High Energy Phys. 07 (2018) 144.

[72] A. E. Cárcamo Hernández, S. Kovalenko, J. W. F. Valle, and C. A. Vaquera-Araujo, J. High Energy Phys. 07 (2017) 118.

[73] A. E. Cárcamo Hernández, H. N. Long, and V. V. Vien, Eur. Phys. J. C 78, 804 (2018).

[74] A. E. Cárcamo Hernández, S. Kovalenko, J. W. F. Valle, and C. A. Vaquera-Araujo, J. High Energy Phys. 02 (2019) 065.

[75] A. E. Cárcamo Hernández and S. F. King, arXiv:1903.02565.

[76] A. E. Cárcamo Hernández, Y. Hidalgo Velásquez, and N. A. Pérez-Julve, Eur. Phys. J. C 79, 828 (2019).

[77] C. Dib, S. Kovalenko, I. Schmidt, and A. Smetana, AIP Conf. Proc. 2165, 020023 (2019).

[78] A. E. Cárcamo Hernández, L. T. Hue, S. Kovalenko, and H. N. Long, arXiv:2001.01748.

[79] R. M. Schabinger and J. D. Wells, Phys. Rev. D 72, 093007 (2005).

[80] B. Patt and F. Wilczek, arXiv:hep-ph/0605188.

[81] A. E. Cárcamo Hernández, S. Kovalenko, R. Pasechnik, and I. Schmidt, J. High Energy Phys. 06 (2019) 056.

[82] D. Bertolini and M. McCullough, J. High Energy Phys. 12 (2012) 118.

[83] T. Robens and T. Stefaniak, Eur. Phys. J. C 75, 104 (2015).

[84] A. Falkowski, C. Gross, and O. Lebedev, J. High Energy Phys. 05 (2015) 057.

[85] M. Gorbahn, J. M. No, and V. Sanz, J. High Energy Phys. 10 (2015) 036.

[86] D. Buttazzo, F. Sala, and A. Tesi, J. High Energy Phys. 11 (2015) 158.

[87] P. P. Giardino, K. Kannike, I. Masina, M. Raidal, and A. Strumia, J. High Energy Phys. 05 (2014) 046.
[88] A. Falkowski, F. Riva, and A. Urbano, J. High Energy Phys. 11 (2013) 111.

[89] P. F. de Salas, D. V. Forero, C. A. Ternes, M. Tortola, and J. W. F. Valle, Phys. Lett. B 782, 633 (2018).

[90] I. Esteban, M. C. Gonzalez-Garcia, A. HernandezCabezudo, M. Maltoni, and T. Schwetz, J. High Energy Phys. 01 (2019) 106.

[91] G. C. Branco, J. M. Gerard, and W. Grimus, Phys. Lett. 136B, 383 (1984).

[92] M. C. Chen and K. T. Mahanthappa, arXiv:1107.3856.

[93] G. Bhattacharyya, I. de Medeiros Varzielas, and P. Leser, Phys. Rev. Lett. 109, 241603 (2012).

[94] I. Girardi, A. Meroni, S. T. Petcov, and M. Spinrath, J. High Energy Phys. 02 (2014) 050.

[95] I. de Medeiros Varzielas and D. Pidt, J. Phys. G 41, 025004 (2014).

[96] M. C. Chen, M. Fallbacher, K. T. Mahanthappa, M. Ratz, and A. Trautner, Nucl. Phys. B883, 267 (2014).

[97] G. C. Branco, I. de Medeiros Varzielas, and S. F. King, Phys. Rev. D 92, 036007 (2015).

[98] A. Gando et al. (KamLAND-Zen Collaboration), Phys. Rev. Lett. 117, 082503 (2016); 117, 109903 (2016).

[99] C. E. Aalseth et al. (Majorana Collaboration), Phys. Rev. Lett. 120, 132502 (2018).

[100] C. Alduino et al. (CUORE Collaboration), Phys. Rev. Lett. 120, 132501 (2018).

[101] J. B. Albert et al. (EXO Collaboration), Phys. Rev. Lett. 120, 072701 (2018).

[102] C. Alduino et al. (CUORE Collaboration), Eur. Phys. J. C 77, 532 (2017).

[103] J. B. Albert et al. (EXO-200 Collaboration), Phys. Rev. D 90, 092004 (2014).

[104] R. Arnold et al. (NEMO-3 Collaboration), Phys. Rev. D 95, 012007 (2017).

[105] I. Abt et al., arXiv:hep-ex/0404039.

[106] T. Gilliss et al. (MAJORANA Collaboration), Int. J. Mod. Phys. Conf. Ser. 46, 1860049 (2018).

[107] A. Blondel et al. (FCC-ee Study Team), Nucl. Part. Phys. Proc. 273-275, 1883 (2016). 